\title{
ESTIMATING PATH LOSS IN WIRELESS LOCAL AREA NETWORKS USING ORDINARY KRIGING
}

\author{
Abdullah Konak \\ Pennsylvania State Univeristy Berks \\ Information Sciences and Technology \\ Reading, PA USA
}

\begin{abstract}
This paper introduces ordinary kriging as a new tool to predict network coverage in wireless local area networks. The proposed approach aims to reduce the cost of active site surveys by estimating path loss at points where no measurement data is available using samples taken at other points. To take the effect of obstacles on the covariance among points into account, a distance measure is proposed based on an empirical path loss model. The performance of the proposed approach is tested in a simulated wireless local area network. The results show that ordinary kriging is able to estimate path loss with acceptable error levels.
\end{abstract}

\section{INTRODUCTION}

Designing a wireless local area network (WLAN) involves several steps, including the selection of Access Point (AP) locations and frequency/power assignments. Network performance indicators such as data rate, packet loss, and jitter at a particular point in the target area of a WLAN depend on the received wireless signal strength at the point. A WLAN is expected to provide $100 \%$ coverage with signal strength above a minimum threshold value over all its target area. To ensure an acceptable level quality of service for users of a WLAN, network designers rely on site survey techniques and/or signal propagation models.

Site surveying for a new WLAN deployment usually starts with placing APs at preliminary locations. Signal strength and other service quality data are then gathered for a set of test points. This survey data is used to modify AP locations to ensure adequate coverage for users in the target area of service. The number and distribution of such test points depend upon the size of the service area as well as its physical topology and anticipated number of users. Proper selection of preliminary AP locations is also important for an effective site survey and design (Hills 2001). There are difficulties associated with data gathering as well. Some parts of the target area might be inaccessible during the survey. Changes in environment may affect quality of measurements and cause variations (Zvanovec, Pechac and Klepal 2003). Site survey personnel must be experienced in carrying out complex site surveys and correctly interpreting the results. Therefore, site surveying is a very time consuming and labor intensive process. Several tools have been developed to aid site surveys and automate the design process based on preexisting site surveys. For example, Rollabout (Hills and Schlegel 2004, Hills, Schlegel, and Jenkins 2004) is a rolling cart with a laptop computer that automatically collects data and creates the coverage map of a WLAN. Commercial site survey software, such as Ekahau (Badman 2006), provide an array of effective tools to survey and plan WLANs.

If APs are not available to gather test point data, modeling tools are utilized for predicting network coverage over a target area. In this approach, the network coverage is simulated using electromagnetic wave propagation models. Comprehensive surveys on electromagnetic wave propagation models for 


\section{Konak}

wireless networks are given in (Sarkar et al. 2003, Zvanovec, Pechac and Klepal 2003). Simulation provides a cost-effective way to analyze alternative design configurations. In addition, simulation can be used to determine preliminary locations of APs before a site survey. However, the accuracy of a coverage prediction depends upon the propagation model and a detailed and accurate representation of the target area. Most modern simulation software are capable of reading maps or blueprints and enable users to define objects on the map of a target area. However, creating a realistic blueprint of the target area including walls, wall types, furniture, stairs and elevator shafts can be costly and time consuming. In addition, simulation results should be verified in a site survey before the final implementation.

In this paper, an ordinary kriging-based empirical approach is proposed to estimate the signal strength in WLANs. The main objective is to create an accurate and complete network coverage map of a WLAN from a limited number of test point measurements. Therefore, the cost of time consuming site surveys can be reduced. In addition, the proposed approach can be used to estimate the network coverage where samples could not be taken due to inaccessibility. Finally, the proposed approach can also be used to validate the accuracy of measurements during a site survey.

There has been limited work in the literature to estimate the network coverage in wireless networks using empirical approaches. In Nasereddin, Konak and Bartolacci (2005), a radial basis function artificial neural network (ANN) is proposed to estimate the signal-to-noise ratio, which is an important indicator for quality of service in cellular wireless networks. To predict the signal-to-noise ratio at a point $p$, this ANN approach utilizes three inputs: the $x-y$ coordinates (indices) of point $p$, the index of the transmitter with highest transmitted power at point $p$, and the transmission power. First the ANN is trained for known points, and then the trained ANN is used to predict the signal-to-noise ratio for unknown points on the target area. Neskovic, Neskovic, and Paunovic (2000) propose a backpropagation ANN to predict the wave propagation for indoor environments. In this case, the input of the ANN includes the distance from the transmitter to the point, objects along the straight line drawn from the transmitter to the point, and topological information about the target area. Therefore, this ANN approach aims to replace physical electromagnetic wave propagation models rather than to predict network coverage from empirical data.

Chen and Kobayashi (2002) propose a linear regression approach to determine the parameters of wave propagation models for WLANs based on the measured signal strengths at test points. The fitted regression model is used to the estimate signal strengths for unknown points. Chen and Kobayashi (2002) report that the quality of the estimation depends on the underlying wave propagation model. On the other hand, the kriging approach proposed in this paper does not assume an underlying wave propagation model, and estimations are based only on field measurements. In a recent paper, Konak (2009) reports that ordinary kriging is competitive with radial basis ANNs to estimate the signal-to-noise ratio in cellular wireless networks, particularly in cases with limited number of sample points available. This paper extends the ordinary kriging approach proposed in (Konak 2009) by considering path loss due to obstacles and other factors in indoor environments. Because WLANs are mainly used in indoors, the attenuation in signal strength due to obstacles, such as walls, building structures and large furniture, is significant. Therefore, obstacles in the environment must be incorporated into estimation. However, this is not possible in the kriging approach proposed in (Konak 2009). To take obstacles into account, a new distance measure is proposed based on an empirical path loss model.

The paper is organized as follows. In Section 2, general path loss models are briefly introduced. Section 3 outlines the ordinary kriging. Section 4 presents the formulated estimation problem and the proposed approach. In Section 5, computational results are presented using a simulated WLAN.

\section{SITE SURVEY AND PATH LOSS MODELS}

Path Loss $(L)$ is a measure of the reduction in power density of an electromagnetic wave as it propagates through space. Path loss occurs because of many reasons, such as free-space-loss, absorption, and diffraction etc. In wireless communications, path loss is usually expressed in decibels $(\mathrm{dB})$ as follows: 


$$
\begin{array}{r}
\text { Konak } \\
L_{d B}=10 \log _{10} \frac{P_{t}}{P_{r}}
\end{array}
$$

where $P_{t}$ and $P_{r}$ are the transmitted and received signal power, respectively. In a WLAN that meets quality-of-service requirements and performs correctly with expected data rate, a minimum level of $P_{r}$ should be ensured at each point over the service area of the network. Therefore, accurately measuring or predicting $L_{d B}$ is an important concern in a WLAN design process.

In addition to site survey, path loss can be predicted using several empirical path loss models. Empirical models to predict path loss rely on average path losses measured for typical types of radio frequencies in various environments. For example, the Okumura model (Okumura and Ohmori 1967; Okumura et al. 1968) and the Hata model (Hata 1980) were developed based on empirical data measured in several urban areas in Japan to predict path loss of terrestrial microwave signals in urban environments. Interested readers might refer to a comprehensive literature survey on empirical path loss models by Sarkar et al. (2003). The most general empirical model for path loss is given as follows (Andersen, Rappaport, and Yoshida 1995):

$$
L_{1}(d)=L_{0}+10 c \log _{10}(d)
$$

where $L_{0}$ is called reference point loss and represents the loss value at one meter(m) distance away from the transmitter, $c$ is the path loss exponent depending on the environment, and $d$ is the Euclidian distance (in $\mathrm{m}$ ) from the transmitter. Parameters $L_{0}$ and $c$ have been determined for various environments through empirical studies (see Zvanovec, Pechac and Klepal 2003) for possible values of $L_{0}$ and $c$ in various environments.

Predicting path loss for indoors, which is the focus in this paper, is more challenging than for outdoors because the variability in the environment is much greater in short distances, and layout of rooms, hallways, furniture as well as various construction materials create complex multipath relationships. When electromagnetic signals pass through walls or floors, they attenuate at significant levels. The path loss due to walls can be taken in to account by considering each wall between a receiver point and a transmitter as follows (Cheung et al. 1998):

$$
L_{2}(d)=L_{1}(d)+\sum_{r \in W} L_{r}
$$

where $W$ is the set of the walls between the receiver and transmitter, $L_{r}$ is the path loss factor $(\mathrm{dB})$ related with wall $r$. For example, the path loss due to a typical dry wall is about $5.4 \mathrm{~dB}$ (see (Anderson et al. 2002, Anderson and Rappaport 2004) for path loss values of different wall and material types). The empirical model given in (3) is simple to implement and widely used in many real-world cases.

\section{ORDINARY KRIGING}

Kriging was developed by Krige (1951) and Matheron (1963) to accurately predict ore reserves from the samples taken over a mining field. Kriging is an interpolation technique based on the methods of geostatistics. Being concerned with spatial data, geostatistics assumes that there is an implied connection between the measured data value at a point in a space and where the point is located (i.e., each data value is associated with a location in the space). Assume that each point $i$ in space is associated with a value $z_{i}$ of interest. Let $u$ represent a point where value $z_{u}$ is unknown (i.e., no sample is available at point $u$ ) and let $V(u)=\left\{1, \ldots, N_{u}\right\}$ be the set of points in the neighborhood of point $u$ such that value $z_{i}$ is known for each point $i \in V(u)$. In ordinary kriging, the most commonly used type of kriging, unknown value $z_{u}$ at a point $u$ is estimated as a weighted-linear combination of the known values in $V(u)$ as follows (Issaks and Srivastava 1989):

$$
\hat{z}_{u}=\sum_{i \in V(u)} w_{i} z_{i}
$$




\section{Konak}

where $\sum_{i \in V(u)} w_{i}=1$.

Kriging is used to determine the optimal weights, which produce the minimum estimation error, in equation (4). Weights are calculated as follows:

$$
\left(\begin{array}{c}
w_{1} \\
\vdots \\
w_{N_{u}} \\
\lambda
\end{array}\right)=\left(\begin{array}{llll}
\gamma\left(h_{1,1}\right) & \ldots & \gamma\left(h_{1, N_{u}}\right) & 1 \\
\vdots & \ddots & \vdots & 1 \\
\gamma\left(h_{N_{u}, 1}\right) & \ldots & \gamma\left(h_{N_{u}, N_{u}}\right) & 1 \\
1 & \ldots & 1 & 0
\end{array}\right)^{-1}\left(\begin{array}{c}
\gamma\left(h_{1, u}\right) \\
\vdots \\
\gamma\left(h_{N_{u}, u}\right) \\
1
\end{array}\right)
$$

where $\gamma\left(h_{i, j}\right)$ is a semivariogram which is a function of distance $h_{i, j}$ between points $i$ and $j$, and $\lambda$ is the Lagrange multiplier to minimize the kriging error. A semivariogram represents the spatial covariance between points in space. According to geostatistics, as distance $h_{i, j}$ between two points $i$ and $j$ increases, the correlation between those points is expected to decrease (i.e., $\operatorname{Cov}\left(z_{i}, z_{j}\right) \leq \operatorname{Cov}\left(z_{i}, z_{k}\right)$ if $\left.h_{i, j} \leq h_{i, k}\right)$. This assumption holds in many real-world cases. For example, water pollutant levels in samples taken in close proximity are expected to be more correlated than in samples taken distance apart.

Ordinary kriging assumes that the mean is constant in the local neighborhood of a point. Therefore, the expected value of estimation error at an unknown point $u$ is zero (i.e., $\left.E\left(\hat{z}_{u}-z_{u}\right)=0\right)$. The weights determined by (5) are called optimal since they minimize the variance of estimation error (i.e., $\left.\operatorname{Var}\left(\hat{z}_{u}-z_{u}\right)\right)$.

Prior to determining the weights using (5), a meaningful distance measure and semivariogram function should be selected. In ordinary kriging, a successful estimation depends on the choice of the semivariogram function. Although there are an infinite number of possible semivariogram functions, most commonly used semivariogram models, such as linear, exponential, and spherical models, provide good results for most data sets. For example, the exponential semivariogram model is given as follows (Bailey and Gatrell 1996):

$$
\gamma\left(h_{i, j}\right)= \begin{cases}0 & h_{i, j}=0 \\ C_{0}+\left(C_{1}-C_{0}\right) \exp \left(1-3 h_{i, j} / R\right) & h_{i, j}>0\end{cases}
$$

where $C_{0}$ is the nugget effect, $C_{1}$ is the still parameter, and $R$ is the range parameter. $R$ defines the distance beyond which the correlation between two points is assumed to be essentially zero. The nugget effect represents variability at distances smaller than the typical sample spacing in the data set. Still parameter is the maximum value of the semivariogram function. Selecting a good semivariogram function requires a careful study of the data set and subjective judgment. General guidelines for a good semivariogram selection are given in Bailey and Gatrell (1996).

Kriging has certain advantages over other interpolation techniques. Kriging is an optimal interpolation method because it produces an unbiased estimate with minimum variance. An important concern in interpolation is to choose the best set of available sample points to be interpolated to estimate an unknown point. The strength of kriging lies in the fact that it defines an optimal set of known points to interpolate by adjusting the weights of the known points. Notice that not only the distances between known and unknown points, but also the distances between known points are considered in equation (5). As a result, clustered sample points containing redundant information are given less weight in estimation. Another advantage of kriging is that every estimate has a corresponding kriging standard deviation. Thus, a reliability map of predictions can be produced. Once the weights and $\lambda$ are calculated using equation (5), the variance of an individual estimation $\hat{z}_{u}$ can be calculated as follows:

$$
\sigma_{\hat{z}_{u}}^{2}=\sum_{i \in V(u)} w_{i} \gamma\left(h_{i, u}\right)+\lambda
$$




\section{Konak}

\section{PROPOSED ORDINARY KRIGING APPROACH TO PATH LOSS ESTIMATION}

The aim of the proposed approach in this paper is to create an accurate and complete network coverage map of a WLAN from a limited number of test point measurements. Let $P$ denote a set of surveyed test points during a site survey. For each point $i \in P$, let $z_{i}$ and $\left(x_{i}, y_{i}\right)$ denote the measured path loss and the $x y$ coordinates of the point, respectively. The problem is to estimate the path loss at a point $u$ where a measurement was not taken. We propose a procedure based on ordinary kriging to estimate the path loss at a point $u$ as follows:

(i). Define a neighborhood of point $u$ in the $x y$ plane and identify the surveyed points in this neighborhood. In this paper, $N$-nearest surveyed points are used as the neighborhood of point $u$. Let $V(u)$ be the set of $N$ surveyed points which are closer to point $u$ than other points in set $P$.

(ii). Define a distance measure and calculate the distances and semivariogram values among the points in $V(u)$ including point $u$.

(iii). Calculate the optimal weights using equation (5).

(iv). Estimate $z_{u}$ using equation (4) and calculate the variance of the estimation using equation (7).

In Step (i), set $V(u)$ can be determined in various ways. In this paper, one of the reasons for using $N$ nearest neighbors approach is to study the effect of $N$ on the estimation error. The Euclidian distance is commonly used as the distance measure in ordinary kriging, and it can also be used in Step (ii). In indoor WLANs, however, the covariance between two points may not solely depend on the distance between points but also on the obstacles between them. For example, two points close to one another may have very different path loss values if there is thick concrete wall between them. To take the effect of walls and other obstacles on the spatial covariance between two points into account, we propose a distance measure based on equation (3). Note that equation (3) is intended to predict the path loss between a transmitter and a receiver, and its unit is $\mathrm{dB}$. In this paper, the distance between two points $i$ and $j$ is defined as follows:

$$
h_{i, j}=\sqrt{\left(x_{i}-x_{j}\right)^{2}+\left(y_{i}-y_{j}\right)^{2}}+10^{E}
$$

where $E=(10 c)^{-1} \sum_{r \in W_{i, j}} L_{r}$ and $W_{i, j}$ represents the set of obstacles between points $i$ and $j$. The first part of equation (8) is the Euclidean distance between points $i$ and $j$. The second part expresses the path loss due to the obstacles in terms of the Euclidian distance. For example, assume that the path loss factor of a wall between two points is $5 \mathrm{~dB}$ and the free space parameter $c$ is 2 for the environment in which the wall resides. The path loss between these two points due to this wall is equal to the free space path loss of $1.778 \mathrm{~m}\left(\right.$ i.e., $\left.10^{(5 /(10 \times 2))}\right)$. Therefore, equation (8) will increase the distance between these two points by $1.778 \mathrm{~m}$.

In this paper, the exponential semivariogram model given in equation (6) is used with parameters $C_{0}=1, C_{1}=10$, and $R=100$. Because the power of electromagnetic waves significantly attenuates at first several meters, the exponential semivariogram model is a good fit. By setting $R=100$, it is assumed that the spatial correlation between two points that are $100 \mathrm{~m}$ apart is zero. Although the range of an AP depends on many factors, $100 \mathrm{~m}$ is usually assumed as the maximum range of a typical AP. By setting $C_{0}=1$ and $C_{1}=10$, it is assumed that the maximum semivariogram value is 10 times more than its minimum value. Because $\log _{10}$ is used in equation (2), the slope of path loss function is smoother compared to corresponding change in the distance. Therefore, a small range is preferred for the semivariogram function. Note that in this paper simulated data is used to test proposed approach, which justifies the selected parameter values of the exponential semivariogram model based on the knowledge of the underlying system. In real-world data, however, the parameters of a semivariogram model should be fitted based on empirical data. 


\section{EXPERIMENTAL STUDY}

To demonstrate the effectiveness of ordinary kriging in estimating path loss in WLANs, the electromagnetic wave propagation in a WLAN with three APs was simulated over an area of $100 \times 100 \mathrm{~m}^{2}$ including different types of walls as shown in Figure 1. To generate a data set, the area was divided into $50 \times 50$ grids and path loss values were sampled at all intersection points of the grids (a total of 2601 data points). Then, the data points were randomly divided into two sets as a training set (i.e., the set of points where the path loss value is assumed to be surveyed) and a test set (i.e., the set of points where the path loss value is not known). Let $\rho$ be the probability of selecting a data point as a training point while randomly partitioning data points into training and test sets. For example, a set of random training points are marked by (*) on the area in Figure 1 for $\rho=.05$. The goal is to estimate the path loss values for test points using training points.

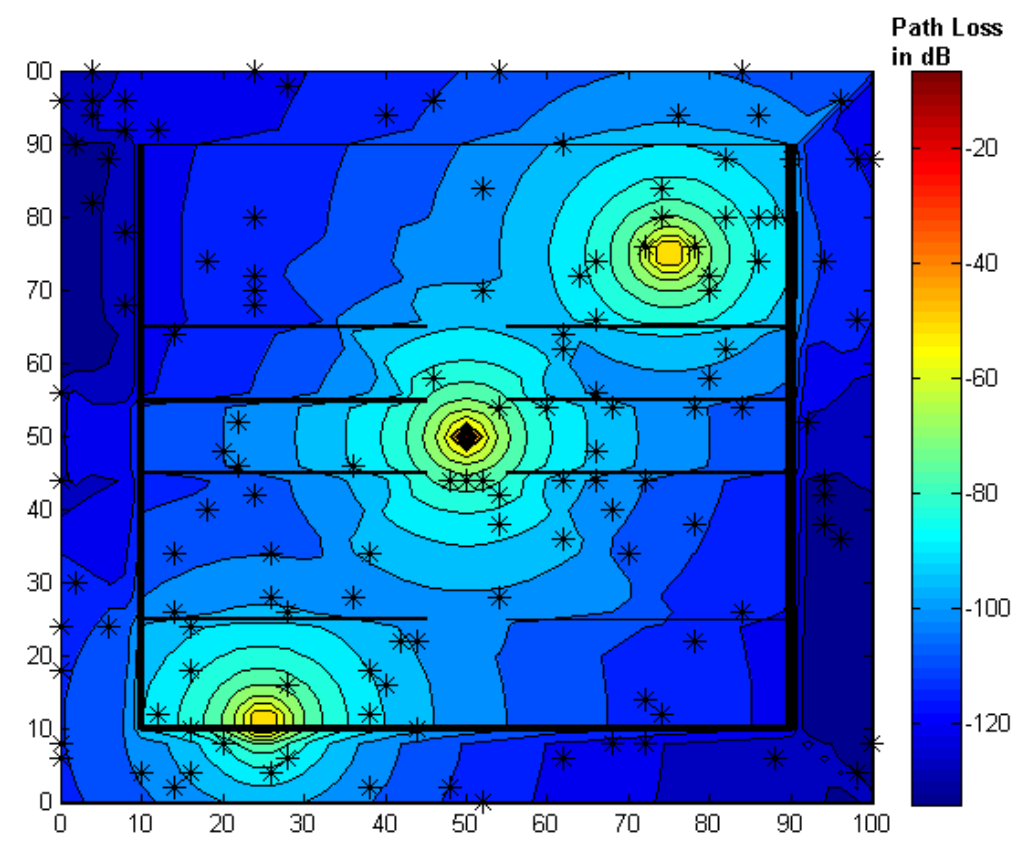

Figure 1: Simulated WLAN coverage over $100 \times 100 \mathrm{~m}^{2}$ area. The area was divided into $50 \times 50$ grids. The path loss values at the corners of the grids were calculated using the empirical model in equation (3) with parameters: $L_{0}=40.2, c=4.2$, and $L_{r}$ ranges from 3 to 15 . Walls are shown by back lines, and the thickness of a wall indicates its path loss factor. Training points that are randomly selected with $\rho=.05$ are marked by (*).

This paper proposes a distance measure by equation (8) to take the effect of obstacles on the spatial covariance between two points into account. It is hypothesized that the proposed distance measure is expected to improve estimation accuracy. Therefore, in the first experimental study, the proposed distance measure was compared to the Euclidean distance for different levels of $\rho$ ranging from 0.02 to 0.1 . For each level of $\rho, 20$ random sets of training and test points were generated, and for each random set of training and test points, the path loss values at test points were estimated from 5-nearest training points using the kriging procedure with two different distance measures (Euclidian distance and equation (8)) as described in Section 4. The Mean Squared Errors (MSE) of estimated values by the two distance measures were compared using a two-way Analysis of Variance (ANOVA) model including the distance measures and $\rho$ as the factors. The result of the ANOVA showed that the proposed distance measure yielded significantly smaller estimation errors compared to Euclidian distance (the $p$-value of the distance measure factor was 0). Figure 2 illustrates the box plots of the Mean Absolute Errors (MAEs) of esti- 


\section{Konak}

mated values by the two distance measures for different levels of parameter $\rho$ in order to demonstrate how much estimated values are close to the actual values. As seen in Figure 2, the proposed distance measure in this paper is effective in reducing the estimation error. Even for small $\rho=0.02$, MAE is less than $5 \%$, and the estimation accuracy improves as more training points are used.

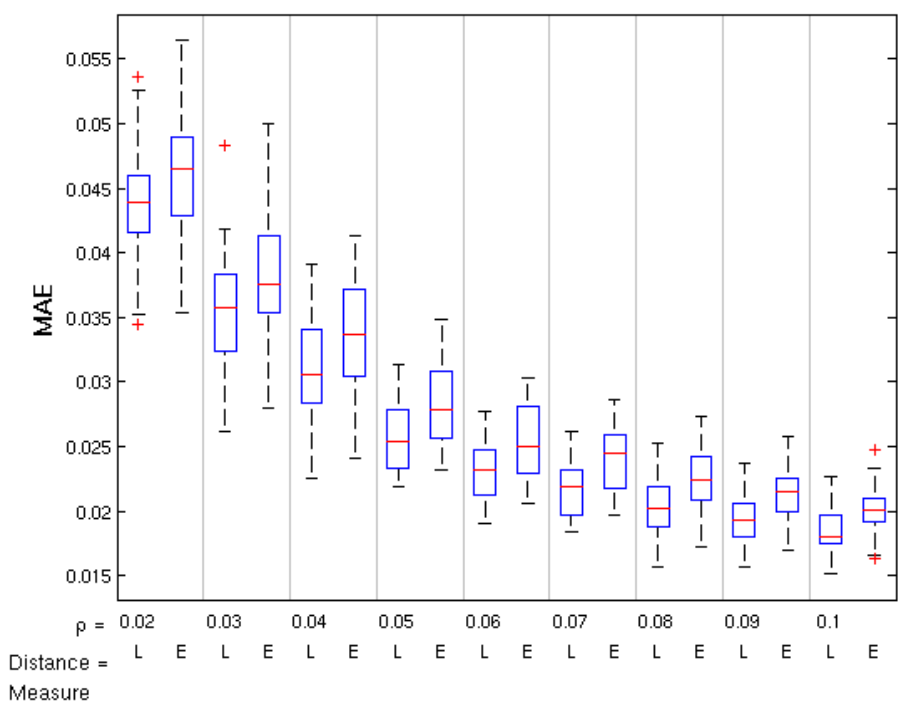

Figure 2: Box-plots of MAEs achieved by (E)-Euclidian distance measure and (L)-the distance measure proposed by equation (8) in this paper. Parameter $\rho$ indicates the probability of selecting a point in the area as a training point. For each level of $\rho$, the simulated data was partitioned into 20 random training and test sets. MAEs were calculated based on test sets.

In the second experimental study, the effect of the neigborhood size ( $N$ of $N$-nearest neighbor) was studied. Figure 3 illustrates the observed MAE values with diffent levels of $N$ for $\rho=0.05$. Initially, MAE quickly improved with the inclusion of a few nearest neighbors, and then it remained steady as additional but farther points were included. This result demonstrates an advantage of kriging- it determines an optimal set of known points to interpolate an unknown point by adjusting the weights of the known points in equation (4). Increasing the neigborhood size in the $N$-nearest neighbor approach may cause clustering of training points. However, kriging sucessfully compensates for the negative effects of this clustering by assigning individual points within a cluster less weight than isolated points.

\section{CONCLUSIONS}

This paper proposes ordinary kriging to predict network coverage in WLANs based on available samples taken in an active site survey. To the best of the author's knowledge, this paper is the first implementation of kriging as a tool in WLANs network survey and design. The objective of the proposed approach is to reduce the cost of time consuming and labor intensive site surveys. The proposed approach can also be used to validate samples taken during a site survey. In addition, a distance measure is proposed to count the effect of obstacles on the spatial covariance among points. This distance measure has been shown to be effective. The proposed approach can be easily embedded within a site survey computer program to interpolate signal coverage for points which are not surveyed in the target area. As further research, it will be interesting to compare the proposed kriging approach with other approaches such as artificial neural networks. 


\section{Konak}

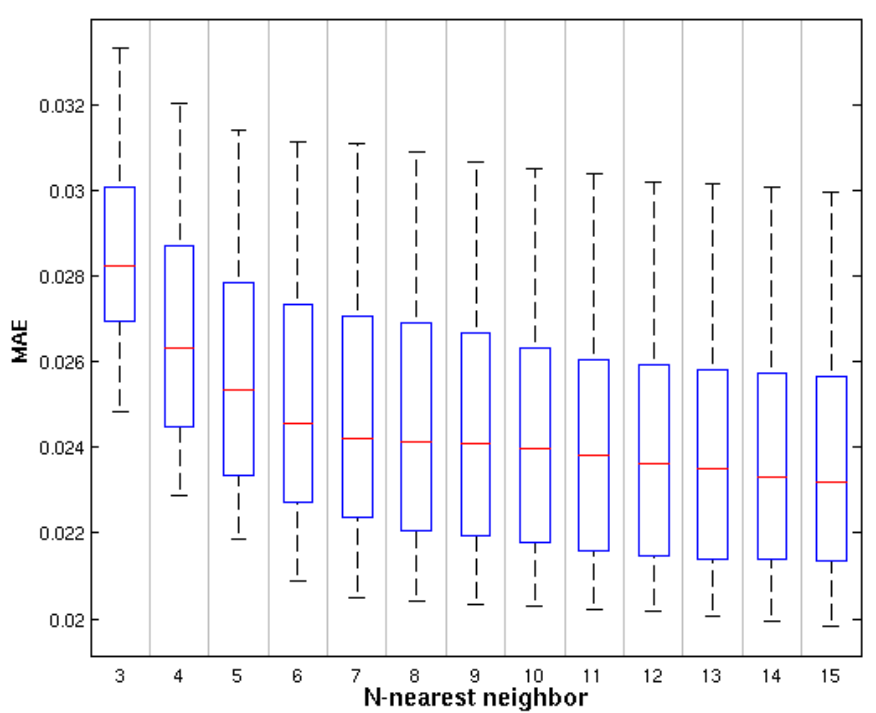

Figure 3: The effect of the neighborhood size on the estimation accuracy for $\rho=0.05$.

\section{REFERENCES}

Andersen, J. B., T. S. Rappaport, and S. Yoshida. 1995. Propagation measurements and models for wireless communications channels. IEEE Communications Magazine 33: 42-49.

Anderson, C. R., and T. S. Rappaport. 2004. In-building wideband partition loss measurements at 2.5 and $60 \mathrm{GHz}$. IEEE Transactions on Wireless Communications 3: 922-8.

Anderson, C. R., T. S. Rappaport, K. Bae, A. Verstak, N. Ramakrishnan, W. H. Tranter, C. A. Shaffer, and L. T. Watson. 2002. In-building wideband multipath characteristics at 2.5 and $60 \mathrm{GHz}$. Proceedings of the IEEE 56th Vehicular Technology Conference 1 :97-101

Badman, L. 2006. Visualize your WLAN [Ekahau's Site Survey 2.2]. Network Computing 17: 38-9.

Bailey, T., and T. Gatrell. 1996. Interactive Spatial Data Analysis Prentice Hall.

Chen, Y., and H. Kobayashi. 2002. Signal strength based indoor geolocation, New York, NY, United states, Institute of Electrical and Electronics Engineers Inc.

Cheung, K. W., J. H. M. Sau, and R. D. Murch. 1998. A new empirical model for indoor propagation prediction. IEEE Transactions on Vehicular Technology 47: 996-1001.

Hata, M. 1980. Empirical formula for propagation loss in land mobile radio services. IEEE Transactions on Vehicular Technology 29: 317-25.

Hills, A. 2001. Large-scale wireless LAN design. IEEE Communications Magazine 39: 98-107.

Hills, A., and J. Schlegel. 2004. Rollabout: a wireless design tool. IEEE Communications Magazine 42: 132-138.

Hills, A., J. Schlegel, and B. Jenkins. 2004. Estimating signal strengths in the design of an indoor wireless network. IEEE Transactions on Wireless Communications 3: 17-19.

Issaks, E. H., and R. M. Srivastava. 1989. An Introduction to Applied Geostatistics. Oxford University Press.

Konak, A. 2009. A kriging approach to predicting coverage in wireless networks. International Journal of Mobile Network Design and Innovation 3: 64-70.

Krige, D. G. 1951. A statistical approach to some basic mine valuation problems on the Witwatersrand. Journal of the Chemical, Metallurgical and Mining Society of South Africa 52: 119-139.

Matheron, G. 1963. Principles of geostatistics. Economic Geology 58: 1246-1266. 
Nasereddin, M., A. Konak, and M. R. Bartolacci. 2005. A neural network-based approach for predicting connectivity in wireless networks. International Journal of Mobile Network Design and Innovation 1: $18-23$.

Neskovic, A., N. Neskovic, and D. Paunovic. 2000. Indoor electric field level prediction model based on the artificial neural networks. IEEE Communications Letters 4: 190-2.

Okumura, Y., and E. Ohmori. 1967. Field strength and its variability in land mobile radio propagation. Electronics and Communications in Japan 50: 69-79.

Okumura, Y., E. Ohmori, T. Kawano, and K. Fukuda. 1968. Field strength and its variability in VHF and UHF land-mobile radio service. Review of the Electrical Communication Laboratory (Tokyo) 16: 825-873.

Sarkar, T. K., J. Zhong, K. Kyungjung, A. Medouri, and M. Salazar-Palma. 2003. A survey of various propagation models for mobile communication. IEEE Antennas and Propagation Magazine 45: 5182.

Zvanovec, S., P. Pechac, and M. Klepal. 2003. Wireless LAN networks design: site survey or propagation modeling? Radioengineering 12: 42-9.

\section{AUTHOR BIOGRAPHY}

ABDULLAH KONAK is an Associate Professor of Information Sciences and Technology at the Pennsylvania State University Berks. He received his degrees in Industrial Engineering; a B.S. from Yildiz Technical University, Turkey, an M.S. from Bradley University, and a Ph.D. from University of Pittsburgh. His current research interest is in the application of Operations Research techniques to complex problems, including such topics as telecommunication network design, network reliability analysis/optimization, facilities design, and data mining. He has published papers in IIE Transactions, Operations Research Letters, OMEGA- The International Journal of Management Science, IEEE Transactions on Reliability, International Journal of Modeling and Simulation, International Journal of Production Research, Engineering Optimization, and Journal of Intelligent Manufacturing. He is a member of IIE and INFORMS. His email address is $<$ konakepsu. edu $>$. 